\title{
Clinical Analysis of 16 Distant Metastatic Retinoblastoma Cases with Event-Free Survival
}

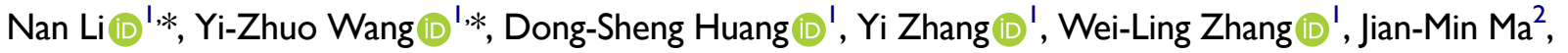 \\ Yan Zhou (D', Ting-Ting Liu' \\ 'Department of Pediatrics, Beijing Tongren Hospital, Capital Medical University, Beijing, 100I76, People's Republic of China; ${ }^{2}$ Department of \\ Ophthalmic Oncology, Beijing Tongren Hospital, Capital Medical University, Beijing, People's Republic of China \\ *These authors contributed equally to this work \\ Correspondence: Dong-Sheng Huang, Department of Pediatrics, Beijing Tongren Hospital, Capital Medical University, No. 2, Xihuan South Road, \\ Yizhuang Economic and Technological Development Zone, Beijing, I00I76, People's Republic of China, Email Hds5I80@sina.com
}

\begin{abstract}
Aim: This study aimed to summarize the clinical characteristics, treatment, and outcomes of distant metastatic retinoblastoma with event-free survival.

Design: Retrospective interventional case series.

Methods: We screened patients with retinoblastoma who survived without events after the comprehensive treatment of distant metastases from June 2015 to February 2021 and collected information regarding their basic characteristics, diagnosis, and treatment. All patients received systemic intravenous chemotherapy. Other treatments included surgical treatment, radiotherapy, intrathecal chemotherapy, and autologous stem cell transplantation.

Results: Among 780 hospitalized patients with retinoblastoma in the pediatric ward, a total of 94 patients with retinoblastoma were diagnosed with distant metastases, and 16 patients with distant metastatic retinoblastoma who survived more than 6 months without events were screened, including eight male and eight female patients. The median age of onset was 29 (range, 11-120) months. Among the 16 patients, central nervous system metastasis (8/16), bone metastasis (8/16), bone marrow infiltration (4/16), lymph node metastasis (4/16), and parotid gland metastasis (3/16) were presented. All patients received treatment for more than 6 months, completed their regimen by February 2021, and survived without events. The median survival time after the onset of retinoblastoma was 50.5 (range, 23-102) months, the median survival time after metastasis was 43.5 (range, 16-71) months, and the median eventfree survival was 29.0 (range, 6-59) months.

Conclusion: Metastatic retinoblastoma may benefit from comprehensive treatments including systemic intravenous chemotherapy and hematopoietic stem cell transplantation. However, recurrence after treatment still needs attention, and patients in complete remission still need long-term follow-up.
\end{abstract}

Keywords: retinoblastoma, distant metastasis, comprehensive treatment

\section{Introduction}

Retinoblastoma (RB) is a malignant tumor of the eye occurring in childhood. The incidence is 1 in 16,000 to 18,000 live births, accounting for approximately $11 \%$ of cancers diagnosed in the first year of life. ${ }^{1-3}$ The goals of treatment for RB include life, globe, and vision salvage. ${ }^{4} \mathrm{RB}$ is considered curable, and improved medical conditions and modern technology have decreased the RB-associated mortality rates in Europe and the US to about 5\% and 3\%, respectively. ${ }^{5}$ However, extraocular dissemination and distant metastasis are important factors leading to poor prognosis of patients with RB, and there are few previous reports of cured cases. Effective treatment of metastatic RB is still a difficult problem in current RB treatment. More effective and standardized treatment methods and clinical guidelines are needed. We summarized the clinical data of 16 patients with metastatic RB who survived without events in Beijing Tongren Hospital, to share our experiences. 


\section{Methods}

The data of patients with RB admitted to the Pediatrics Department of Beijing Tongren Hospital June 2015 to February 2021 were collected. Beijing Tongren Hospital is one of the largest retinoblastoma diagnosis and treatment centers in China. The pediatric department of our hospital admitted patients with advanced RB from all over China. Among 780 hospitalized patients with retinoblastoma in the pediatric ward, a total of 94 patients with retinoblastoma were diagnosed with distant metastases. The inclusion criteria were as follows: 1) patients diagnosed with RB in our hospital with confirmed involvement of tissues other than the eyeball and orbit; 2) after comprehensive treatment in our hospital, the primary and metastatic tumors of the patients achieved complete remission and all patients survived events free more than 6 months by August 2021; and 3) all guardians of participating patients provided written informed consent for treatment. This study complies with the Declaration of Helsinki and this study protocol was approved by the Ethics Committee of Beijing Tongren Hospital, Capital Medical University [TRECKY2021-096].

The exclusion criteria were as follows: 1) irregular treatment after metastasis; 2) treatment had not ended or an eventfree survival of less than 6 months; 3) during the treatment of RB, metastasis occurred due to disease progression; and 4) patients with trilateral RB.

\section{Diagnosis of RB}

All patients completed ocular ultrasound, orbital CT, ocular MRI, fundus examination, and/or pathological examination and were diagnosed as RB. According to the AJCC RB TNM staging system, all patients were in stage cM1a or cM1b.

\section{Diagnosis of RB Metastasis}

Patients completed systemic examinations, including serum neuron-specific enolase (NSE) analysis, bone puncture, lumbar puncture, lymph node ultrasound, cranial MRI, bone scan, PET/CT, or pathological examination. The involvement of tissues other than the eyeball and orbit was confirmed.

\section{Intravenous Chemotherapy (IVC)}

All patients with metastasis RB underwent systemic IVC. Commonly used chemotherapy drugs included vincristine (V), etoposide (E), carboplatin (C), cisplatin (P), cyclophosphamide (C), ifosfamide (I), and topotecan (T). Commonly used regimens included carboplatin, etoposide, vincristine (CEV)/ cisplatin, cyclophosphamide, etoposide, vincristine (PCEV)/ cyclophosphamide, carboplatin, etoposide, vincristine (CCEV), and individualized chemotherapy regimens were applied for children with poor treatment effects.

\section{Surgical Treatment}

Eyeball enucleation was performed for late-stage RB eye disease or failed eye-protecting treatment. Orbital exenteration was performed for children with extraocular orbital involvement to save lives.

\section{Radiotherapy}

For cases of central nervous system (CNS) metastasis or localized soft tissue/bone metastasis with poor therapeutic effects, radiotherapy was combined with other treatments. Generally, patients who receive radiotherapy are older than 3 years; and the age limit can be relaxed to 2 years old if necessary. The treatment is carried out in the form of fractionated radiotherapy, and the total dose of radiotherapy is generally $36-45 \mathrm{~Gy}$.

\section{Hematopoietic Stem Cell Transplantation}

If the tumor did not reach complete remission (CR) after 4-6 cycles of IVC or combined treatment, autologous stem cell transplantation (ASCT) should be considered. Before treatment, consent shall be obtained from the patient's family, and the tolerance of children's physical condition to treatment needs to be evaluated. 


\section{Intrathecal Chemotherapy}

All patients with CNS metastases or lesions close to the brain were given monthly lumbar punctures and intrathecal chemotherapy (dexamethasone, cytarabine, and methotrexate).

\section{Follow-Up}

All patients were followed up to August 2021 through outpatient review or telephone follow-up. The median follow-up time was 50.5 (range, 23-102) months, The RB overall survival (OS) refers to the diagnosis of retinoblastoma to the end of follow-up; Event-free survival (EFS) after metastasis refers to the time from the diagnosis of RB metastasis and the start of treatment to the occurrence of any events, including death, disease progression, and disease recurrence. EFS after treatment refers to the time from the end of all treatments to the occurrence of any events in patients with metastatic RB, including death, disease progression, and disease recurrence.

\section{Results}

Among 94 patients with retinoblastoma who were diagnosed with distant metastases, a total of 16 patients with RB met the above enrollment criteria up to August 2021, including eight cases with CNS metastasis and eight cases without CNS metastasis. Among them, eight were male, and eight were female. The median age of onset was 29 (range, 11-120) months. No children had a family history of RB. Two cases had binocular disease, 14 cases had monocular disease, and there were 18 diseased eyes. Seven patients had metastatic RB at diagnosis, and the remaining cases had metastatic disease after recurrence. The nine recurrent patients were treated before recurrence by enucleation (7/9), systemic IVC (2/ 13), and other methods, including laser therapy, intra-arterial chemotherapy, vitrectomy. One patient who was misdiagnosed as Coats disease received subretinal fluid drainage combined with intravitreal injection of anti-vascular endothelial growth factor.

The most common sign was leukocoria (9/16), and other manifestations included decreased vision, conjunctival hyperemia, strabismus, and exophthalmos (Table 1). After excluding two patients whose pathological report was lost, the pathological risk factors of other patients were optic nerve invasion (7/14), large choroidal invasion (3/14), and others, including anterior segment, lamina, sclera, and extraocular invasion (Table 1). Among the 16 patients, CNS metastasis (8/ 16), bone metastasis (8/16), bone marrow infiltration (4/16), lymph node metastasis (4/16), parotid gland metastasis $(3 /$ 16) (Figure 1), and orbital involvement (including the optic nerve, 13/16) were presented. Nine patients had a significant increase in the serum NSE level at the time of metastasis. However, the increase in serum NSE was not significant in children with simple bone metastasis and simple optic nerve intracranial invasion.

All patients received systemic IVC after metastasis (Table 2). The chemotherapy cycles after metastasis depended on the condition (metastatic site, tumor load and response to treatment), with a median of 12.5 (range, 8-19) cycles. Common side effects in patients during chemotherapy included bone marrow suppression, liver damage, electrolyte disturbances, and gastrointestinal reactions. Other treatments included surgery, radiation therapy, and autologous stem cell transplantation. Among the 10 patients who underwent surgery, 11 diseased eyes in nine patients underwent eyeball enucleation, and one patient who had eye enucleation before recurrence underwent orbital exenteration. In addition, one patient underwent eyeball enucleation and cranioorbital metastasis resection. After treatment, only one eye with stage $\mathrm{C}$ remained in the 18 diseased eyes, and the eye preservation rate was $5.7 \%$. Seven patients with CNS metastases and one patient with craniofacial bone and sinus metastases received fractionated radiotherapy. The median radiotherapy dose was 45 (range, 36-50) Gy. One recurrent RB patient with bone, lymph node, and bone marrow metastases underwent ASCT and achieved CR. By the end of the follow-up period, the EFS after metastasis was 33 months. Lumbar puncture and intrathecal chemotherapy (range, 6-14 times) were given monthly for all patients with CNS metastasis or lesions close to the brain; other patients undergo intermittent lumbar punctures to assess whether the CNS was involved.

All patients had completed treatment by February 2021, and survived without events. By the end of treatment, the serum NSE levels of all patients were within 10.6-27.3ng $/ \mathrm{mL}$. The median OS of RB was 50.5 (range, 23-102) months, the median EFS after metastasis was 43.5 (range, 16-71) months, the median EFS after treatment was 29 (range, 6-59) months (Figure 2), six patients (6/16) achieved an EFS of more than 3 years after all treatments ended. Among all 94 
Table I Characteristics of Included Patients

\begin{tabular}{|c|c|}
\hline Characteristic & Number (n, \%) \\
\hline Age (years), $<3 / \geq 3$ & $9(56.2) / 7(43.8)$ \\
\hline Gender, M/F & $8(50.0) / 8(50.0)$ \\
\hline Eye affected, Mon/Bin & $14(87.5) / 2(12.5)$ \\
\hline \multicolumn{2}{|l|}{ Presenting signs } \\
\hline Leukocoria & $9(56.2)$ \\
\hline Vision loss & $6(37.5)$ \\
\hline Conjunctival hyperemia & $5(31.3)$ \\
\hline Squint & $3(18.8)$ \\
\hline Proptosis & $3(18.8)$ \\
\hline \multicolumn{2}{|l|}{ Metastasis sites } \\
\hline Bone metastasis & $8(50.0)$ \\
\hline CNS metastasis & $8(50.0)$ \\
\hline Lymph node metastasis & $4(25.0)$ \\
\hline Bone marrow infiltration & $4(25.0)$ \\
\hline Parotid gland metastasis & $3(18.8)$ \\
\hline \multicolumn{2}{|c|}{ Pathologically high-risk site of invasion } \\
\hline Optic nerve invasion & $7(43.8)$ \\
\hline Extensive choroidal invasion & $3(18.8)$ \\
\hline Anterior quarter & $2(12.5)$ \\
\hline Sieve plate & $3(18.8)$ \\
\hline Sclera & $\mathrm{I}(6.25)$ \\
\hline Extraocular involvement & $2(12.5)$ \\
\hline
\end{tabular}

Abbreviations: M, male; F, female; Mon, monocular; Bin, binocular; CNS, central nervous system.

patients with metastatic RB, 65 patients died, including eight patients who died of tumor recurrence after CR; two patients were lost to follow-up; 27 patients survived (four patients were under treatment, and seven patients achieved CR but did not meet the above inclusion criteria). The overall survival rate of 94 patients with metastatic RB was $28.7 \%$ (Figure 3). Of the 90 patients who ended the treatment, 31 patients achieved CR, and eight patients died after recurrence, including one patients who received ASCT. The recurrence rate after CR was $25.8 \%$.

\section{Discussion}

China is the most populous country globally and documents approximately 1000 new cases of RB every year, accounting for $1 / 8$ of all cases worldwide. ${ }^{6}$ An increased number of cases diagnosed in middle and late stages and limited medical resources have resulted in lower eye protection rates and survival rates of RB patients in China compared with those in developed countries. The prognosis remains relatively poor for patients whose disease disseminates into the CNS and those with distant metastatic disease. ${ }^{7}$ The most common metastatic site of RB is the intracranial area, accounting for approximately $50 \%$ of cases. ${ }^{8}$ Bone is the second most common site. In addition, studies have confirmed that RB metastasizes to the parotid glands and submandibular lymph nodes through lymphatic vessels, but there are few previous case reports. ${ }^{9}$

Patients with metastatic RB should undergo a whole-body examination to assess their condition, including a fundus examination, lumbar puncture, bone puncture, MRI of the eye and head, bone scan, and imaging of lymph nodes, lungs, and abdomen (or PET-CT examination). Serum NSE levels can be elevated in some RB patients with metastases and are used as a blood indicator for monitoring. However, they are not sensitive enough in patients with metastases, especially in those with simple bone and optic nerve metastases. During the follow-up period in this study, the serum NSE level of all patients was maintained within $10.6-27.3 \mathrm{ng} / \mathrm{mL}$ at the end of treatment, indicating that the serum NSE level may have biomarker value in the diagnosis and treatment of RB. 


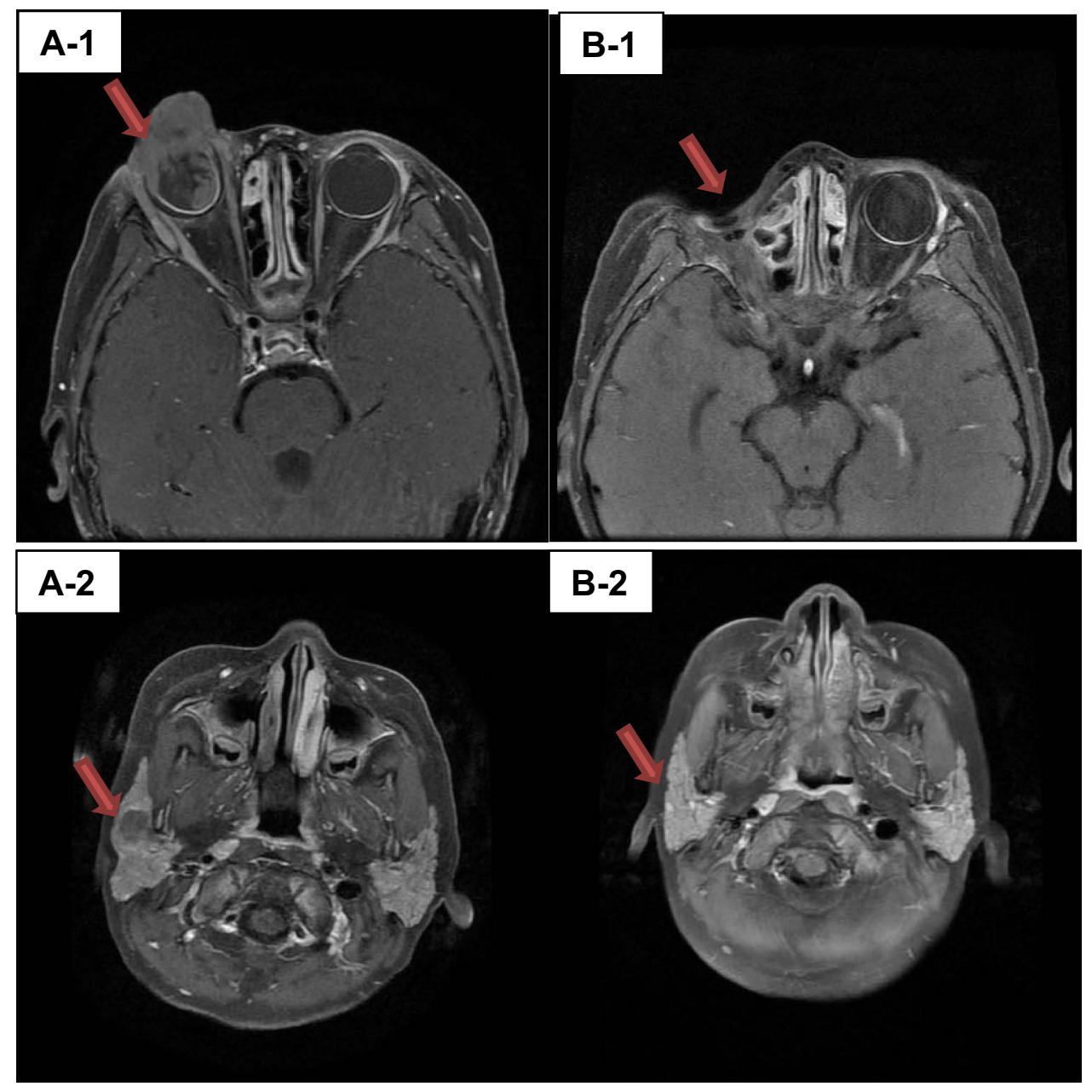

Figure I RB case with metastasis to parotid gland. (A-I) TIWI+C FS Status after vitrectomy (misdiagnosed at onset) in the right eye. Irregular equal TI signals can be seen around the eyeball, locally protruding outside the orbit, and the enhanced scan is slightly enhanced, involving the lacrimal sac area and extraocular muscles. (A-2) $T I W I+C$ FS Abnormal signal in the right parotid area with swollen lymph nodes, and the enhanced scan showed uneven enhancement. (B-I) TIWI+C FS Postoperative state of the right eye, with mild and uneven enhancement of the orbital fat body; (B-2) TIWI+C FS The original parotid gland area and lymph node enlargement subsided.

Patients with RB metastasis have a low survival rate and poor prognosis. RB metastasis is an important cause of death in RB patients. Previously, there have been some reports of successful RB treatment in the metastatic stage. Wang et al., ${ }^{9}$ Purkayastha et al ${ }^{10}$ have reported the successful treatment of RB patients with parotid gland and soft tissue metastasis by systemic IVC combined with local radiotherapy. Wang et al., ${ }^{11}$ Vempuluru et al, ${ }^{12}$ and others reported the use of systemic IVC combined with external radiotherapy for the treatment of RB patients with bone metastasis and CNS metastasis. In a study by Palma et al, ${ }^{13} 11$ patients with RB metastasis were reported to have undergone high-dose chemotherapy (HDC) combined with ASCT, and two children received post-ASCT CNS radiotherapy. Seven children showed disease-free survival, including those with bone metastasis, bone marrow metastasis, optic chiasm masses, and cerebrospinal fluid metastasis. Consequently, they proposed that HDC-ASCT is a feasible and effective treatment for children with metastatic RB. ${ }^{13}$ In our study, we reported 16 patients with metastatic RB, including eight children with CNS metastasis (two cases of brain parenchyma metastasis and six cases of optic nerve and chiasm metastasis) and eight patients with metastases in other tissues (bone, parotid glands, lymph nodes, and bone marrow). All patients achieved CR after treatment and survived event-free during the follow-up period. The overall survival rate of 94 children with RB metastasis was $28.7 \%$; those with event-free survival of more than 6 months who met the enrollment criteria accounted for $17.0 \%$. In summary, although the prognosis is particularly poor, a reasonable selection of comprehensive treatment methods may provide effective treatment for patients with metastatic RB. However, since the recurrence rate of children with $\mathrm{RB}$ who achieved $\mathrm{CR}$ after treatment was as high as $25.8 \%$, long-term follow-up is 
Table 2 Diagnosis and Treatment of Patients with Metastasis

\begin{tabular}{|c|c|c|c|c|c|c|c|c|c|c|c|}
\hline & \multirow{2}{*}{$\begin{array}{l}\text { Age } \\
\text { (Mon) }\end{array}$} & \multirow[t]{2}{*}{ Stage } & \multirow[b]{2}{*}{$\begin{array}{l}\text { Time to } \\
\text { Metastasis }\end{array}$} & \multicolumn{2}{|l|}{ Metastasis Site } & \multicolumn{5}{|c|}{ Treatment after RB Metastasis } & \multirow{2}{*}{$\begin{array}{l}\text { EFS } \\
\text { (Mon) }\end{array}$} \\
\hline & & & & Evidence & Metastatic Sites & $\begin{array}{l}\text { IVC } \\
\text { Cycles }\end{array}$ & $\begin{array}{l}\text { RT } \\
\text { (Gy) }\end{array}$ & Surgery & $\begin{array}{l}\text { Intrathecal } \\
\text { Chemotherapy } \\
\text { (Cycles) }\end{array}$ & $\begin{array}{l}\text { Other } \\
\text { Treatments }\end{array}$ & \\
\hline I & 22 & cMla & Recurrence & $\begin{array}{l}\mathrm{PET} / \mathrm{CT} \text {, } \\
\mathrm{BM} \text { cytology }\end{array}$ & Bone, lymph nodes, bone marrow & 8 & & - & & ASCT & 33 \\
\hline 2 & 27 & cMla & Recurrence & $\begin{array}{l}\mathrm{PET} / \mathrm{CT} \text {, } \\
\mathrm{BM} \text { cytology }\end{array}$ & Bone, lymph nodes, bone marrow & 9 & & - & & & 28 \\
\hline 3 & 28 & cMla & Recurrence & Bone scan, MRI & Bone, sinuses (near intracranial) & 14 & 43.2 & - & 14 & & 37 \\
\hline 4 & 53 & pMla & Recurrence & Pathology, MRI & Orbit, lymph node, parotid gland & II & & Enucleation & & & 42 \\
\hline 5 & 23 & cMla & Recurrence & MRI, bone scan & Bone, orbit & 9 & & Orbital exenteration & & & 71 \\
\hline 6 & 44 & cMla & Onset & Bone scan & Bone, Intraorbital segment of optic nerve & 9 & & Enucleation & 6 & & 64 \\
\hline 7 & 38 & cMla & Onset & Bone scan & Bone, Intraorbital segment of optic nerve & 12 & & Enucleation & 11 & & 45 \\
\hline 8 & 17 & pMla & Recurrence & $\begin{array}{l}\text { MRI, } \\
\text { BM } \\
\text { immunocytology }\end{array}$ & Bone marrow, orbit, parotid gland & 12 & & Enucleation & 6 & & 16 \\
\hline 9 & 27 & cMIb & Recurrence & MRI & $\begin{array}{l}\text { Anterior and middle cranial fossa, } \\
\text { frontotemporal lobe, orbit, bone marrow, } \\
\text { bone, lymph nodes, sinuses }\end{array}$ & 19 & 46 & - & 13 & & 65 \\
\hline 10 & 25 & cMIb & Recurrence & MRI & $\begin{array}{l}\text { Temple lobe, orbits, bones, sinuses, parotid } \\
\text { gland }\end{array}$ & 17 & 45 & - & 11 & & 68 \\
\hline 11 & 120 & cMIb & Onset & MRI & Optic nerve/Optic chiasm & 9 & 45 & Enucleation & 9 & & 47 \\
\hline 12 & 56 & cMIb & Onset & MRI & Intracranial optic nerve & 14 & - & $\begin{array}{l}\text { Enucleation } \\
\text { +cranioorbital } \\
\text { communication mass } \\
\text { resection }\end{array}$ & 9 & & 69 \\
\hline 13 & 11 & cMIb & Recurrence & $\begin{array}{l}\text { MRI, BM } \\
\text { cytology }\end{array}$ & Optic nerve/Optic chiasm & 15 & 36 & & 9 & & 46 \\
\hline 14 & 37 & cMIb & Onset & MRI & Optic nerve/Optic chiasm & 15 & 45 & Enucleation & 14 & & 22 \\
\hline 15 & 82 & cMIb & Onset & MRI & Optic nerve/Optic chiasm & 16 & 43.2 & Enucleation & 14 & & 35 \\
\hline 16 & 30 & cMIb & Onset & MRI & Intracranial optic nerve & 13 & 50.4 & Enucleation & 9 & & 33 \\
\hline
\end{tabular}

Abbreviations: IVC, intravenous chemotherapy; EFS, event-free survival after metastasis; BM, bone marrow; ASCT, autologous stem cell transplantation; RT, radiation Therapy. 


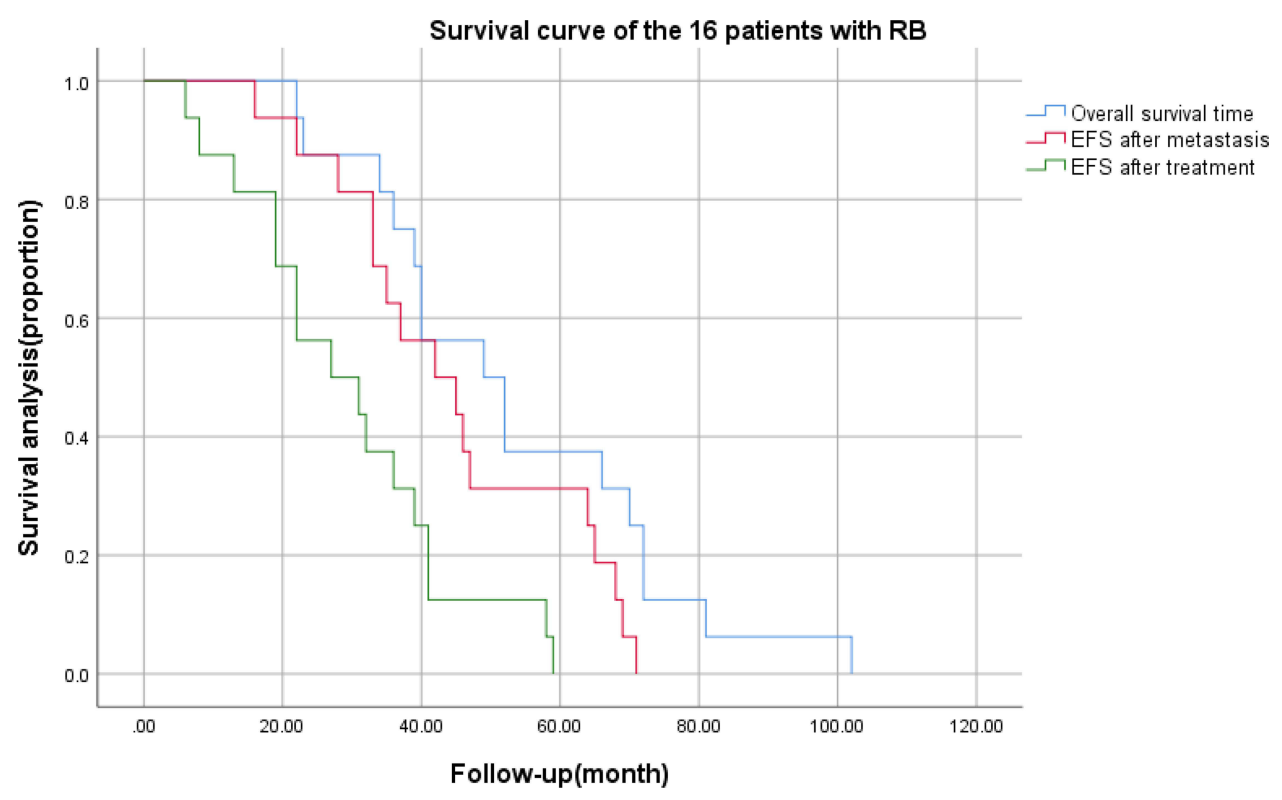

Figure 2 Survival curve of the 16 patients with RB with metastasis. Kaplan-Meier survival curve of the 16 included patients, the median OS of RB was 50.5 (range, 23-102) months, the median EFS after metastasis was 43.5 (range, 16-7I) months, the median EFS after treatment was 29 (range, 6-59) months.

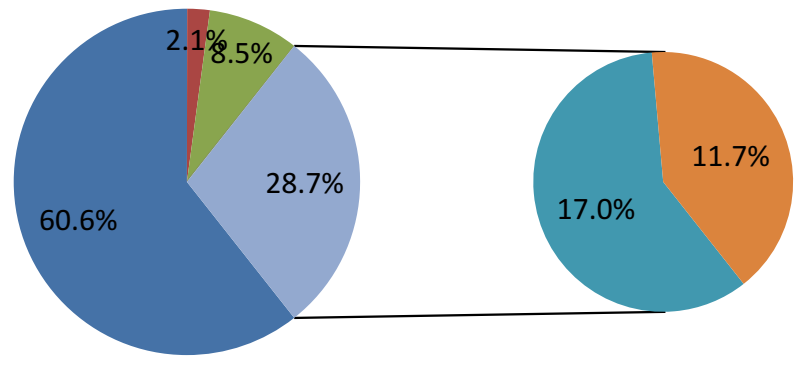

Death after progression
Lost
Recurrence and death after CR
surviving patients
Survived and included
Other surviving patients

Figure 3 RB case with metastasis. Among all 94 patients with metastatic RB, 65 patients died, including eight patients who died of tumor recurrence after CR; two patients were lost to follow-up; 27 patients survived (four patients were under treatment, and seven patients achieved CR but did not meet the above inclusion criteria). The overall survival rate of 94 patients with metastatic RB was $28.7 \%$.

mandatory in all metastatic RB survivors, and it is necessary to be alert to the recurrence of RB, even in patients with CR after ASCT.

On the basis of our experience, systemic IVC is effective for the treatment of metastatic RB. The chemotherapy regimen for the treatment of IVC should be improved on the basis of the traditional CEV regimen. The chemotherapy cycle and regimen should be adjusted based on the patient's condition; patients with CNS metastasis or systemic dissemination, high tumor load, or patients with poor response to treatment usually require more chemotherapy cycles; individualized treatment can be considered for complex cases. For patients with intracranial metastases or lesions close to the brain, combined fractionated radiotherapy (recommended total radiation dose of $45 \mathrm{~Gy}$ ) and intrathecal chemotherapy (recommended 9-12 cycles of intrathecal chemotherapy) are required. Among cases with CNS metastasis, simple intracranial optic nerve infiltration may be the subtype with the best prognosis. For patients with late-stage metastatic $\mathrm{RB}$, eyeball enucleation should be performed as appropriate. For intra-orbital metastases, orbital exenteration can be performed; the lesions should be removed as much as possible, and the eyeballs should not be preserved blindly. For large tumors or extensive lesions, systemic chemotherapy can be administered first for tumor reduction, and then other treatment options may be combined. Autologous stem cell transplantation may be effective for metastatic RB.

At present, the main chemotherapeutic strategy for RB is the CEV regimen. Although this chemotherapy regimen has been proven effective in the treatment of non-metastatic RB patients, it is insufficient in the treatment of advanced 
RB. In recent years, individualized treatment has been increasingly recognized, and we have administered individualized treatment for complex cases. However, new chemotherapeutic drugs and chemotherapy regimens require further research and clinical observation of their efficacy. Molecular targeted drugs have recently been suggested to be more effective and less toxic than traditional treatments, and local administration enhances the effect of drugs, ${ }^{14}$ but there is no effective clinical application. Published literature indicates that HDC-ASCT is an option for the treatment of metastatic and extraocular RB. Long-term survival rates are reported to be $50 \%$ to $75 \%$, but there are still some limitations, such as recurrence after transplantation and the small sample size of existing studies. ${ }^{15,16}$ Furthermore, hematopoietic stem cell transplantation for the treatment of metastatic RB still needs additional clinical research and exploration.

The prognosis of early stage RB cases is good, but the prognosis of metastatic cases is very poor. The high survival rate of $\mathrm{Rb}$ patients in developed countries benefits from the early identification and standardized management of diseases. At present, $\mathrm{Rb}$ has been included in the "national catalogue of rare diseases" in China. As a developing country, the distribution of medical resources in China is unbalanced; ophthalmologists in primary medical institutions should improve the ability to recognize RB disease, and refer patients to regional medical centers for early and standardized treatment as soon as possible. In addition, families with genetic risk of RB need to seek for genetic counseling and early screening of RB disease. Under the current medical situation, early identification of retinoblastoma, standardized treatment and long-term follow-up management are very important to improve the prognosis of $\mathrm{Rb}$.

\section{Conclusion}

The prognosis of distant metastatic RB is particularly poor. Systemic IVC combined with surgery, intrathecal chemotherapy, radiotherapy, hematopoietic stem cell transplantation, and other treatments may be effective in the treatment of metastatic RB. However, distant metastasis of RB is difficult to treatment, and the survival rate is low, recurrence after treatment is also worrying, even ASCT cannot completely avoid RB recurrence. Thus, it remains the main cause of death in children with RB, and patients in complete remission still need long-term follow-up. A larger sample for clinical research and the exploration of new treatment methods are needed.

\section{Synopsis}

We report 16 cases of metastatic retinoblastoma who survived event-free through chemotherapy-based comprehensive treatment. Comprehensive treatment may improve the prognosis of patients with metastatic retinoblastoma.

\section{Abbreviations}

RB, retinoblastoma; NSE, neuron-specific enolase; IVC, intravenous chemotherapy; CNS, central nervous system; CR, complete remission; ASCT, autologous stem cell transplantation; OS, overall survival; EFS, event-free survival; HDC, high-dose chemotherapy.

\section{Supportive Foundations}

The Beijing Hospitals Authority' Ascent Plan (DFL20180201); The Beijing Hospitals Authority' Ascent Plan (DFL20190201).

\section{Data Sharing Statement}

The raw/processed data required to reproduce these findings cannot be shared at this time as the data also forms part of an ongoing study.

\section{Author Contributions}

Nan Li and Yi-zhuo Wang: Collect and organize data, conceive and write articles, Dong-sheng Huang and Yi Zhang: Case analysis and revision of the paper; Jian-Min Ma, Yan Zhou and Ting-Ting Liu: Collection of clinical data; WeiLing Zhang: Review and consent of the article; All authors made a significant contribution to the work reported, whether that is in the conception, study design, execution, acquisition of data, analysis and interpretation, or in all these 
areas; took part in drafting, revising or critically reviewing the article; gave final approval of the version to be published; have agreed on the journal to which the article has been submitted; and agree to be accountable for all aspects of the work.

\section{Disclosure}

Nan Li and Yi-Zhuo Wang are co-first authors for this study. The authors declare that they have no conflicts of interest.

\section{References}

1. Dimaras H, Corson TW. Retinoblastoma, the visible CNS tumor: a review. J Neurosci Res. 2019;97(1):29-44. doi:10.1002/jnr.24213

2. Wong JR, Tucker MA, Kleinerman RA, et al. Retinoblastoma incidence patterns in the US surveillance, epidemiology, and end results program. JAMA Ophthalmol. 2014;132(4):478-483. doi:10.1001/jamaophthalmol.2013.8001

3. Belson PJ, Eastwood JA, Brecht ML, et al. A review of literature on health-related quality of life of retinoblastoma survivors. J Pediatr Oncol Nurs. 2020;37(2):116-127. doi:10.1177/1043454219888805

4. Kaliki S, Vempuluru VS, Priya Y, et al. Risk factors for recurrent retinoblastoma after intravenous chemotherapy. Int Ophthalmol. 2021;41 (6):2033-2039. doi:10.1007/s10792-021-01759-4

5. Tomar AS, Finger PT, Gallie B, et al. A multicenter, international collaborative study for American Joint Committee on cancer staging of retinoblastoma: part I: metastasis-associated mortality. Ophthalmology. 2020;127(12):1719-1732. doi:10.1016/j.ophtha.2020.05.050

6. Gao J, Zeng J, Guo B, et al. Clinical presentation and treatment outcome of retinoblastoma in children of South Western China. Medicine. 2016;95 (42):e5204. doi:10.1097/MD.0000000000005204

7. Doz F, Neuenschwander S, Plantaz D, et al. Etoposide and carboplatin in extraocular retinoblastoma: a study by the Société Française d'Oncologie Pédiatrique. J Clin Oncol. 1995;13(4):902-909. doi:10.1200/JCO.1995.13.4.902

8. Ebata K, Mizutani H, Kaneda T, et al. Metastatic retinoblastoma to the orofacial region. J Oral Maxillofac Surg. 1991;49(10):1120-1123. doi:10.1016/0278-2391(91)90149-G

9. Wang P, Li YJ, Zhang SB, et al. Metastatic retinoblastoma of the parotid and submandibular glands: a rare case report. BMC Ophthalmol. 2017;17 (1):229. doi:10.1186/s12886-017-0627-8

10. Purkayastha A, Sharma N, Pathak A, et al. An extremely rare case of metastatic retinoblastoma of parotids presenting as a massive swelling in a child. Transl Pediatr. 2016;5(2):90-94. doi:10.21037/tp.2016.02.01

11. Vempuluru VS, Jakati S, Kaliki S. Delayed metastasis in patients with intraocular retinoblastoma: a review of three cases. Eur J Ophthalmol. 2021;31(4):2042-2047. doi:10.1177/1120672120946285

12. Wang YZ, Zhang Y, Huang DS, et al. Clinical characteristics, treatment and prognosis of children with unilateral retinoblastoma and intracranial segment of Retrobulbar optic nerve invasion. BMC Ophthalmol. 2021;21(1):38. doi:10.1186/s12886-020-01768-4

13. Palma J, Sasso DF, Dufort G, et al. Successful treatment of metastatic retinoblastoma with high-dose chemotherapy and autologous stem cell rescue in South America. Bone Marrow Transplant. 2012;47(4):522-527. doi:10.1038/bmt.2011.108

14. Kaewkhaw R, Rojanaporn D. Retinoblastoma: etiology, modeling, and treatment. Cancers. 2020;12(8):2304. doi:10.3390/cancers12082304

15. Uppuluri R, Jayaraman D, Sivasankaran M, et al. Successful treatment of relapsed isolated extraocular retinoblastoma in the right fibula with high-dose chemotherapy and autologous stem cell transplantation. J Pediatr Hematol Oncol. 2019;41(5):402-403. doi:10.1097/ MPH.0000000000001462

16. Rastogi N, Kapoor R, Yadav SP. Haploidentical hematopoietic stem cell transplantation for relapsed metastatic retinoblastoma. J Pediatr Hematol Oncol. 2020;42(8):499. doi:10.1097/MPH.0000000000001955

Cancer Management and Research

Dovepress

\section{Publish your work in this journal}

Cancer Management and Research is an international, peer-reviewed open access journal focusing on cancer research and the optimal use of preventative and integrated treatment interventions to achieve improved outcomes, enhanced survival and quality of life for the cancer patient. The manuscript management system is completely online and includes a very quick and fair peer-review system, which is all easy to use. Visit http://www.dovepress.com/testimonials.php to read real quotes from published authors.

Submit your manuscript here: https://www.dovepress.com/cancer-management-and-research-journal 